\title{
Underwater Noise and its Statistical Analysis to Qualify the Signal into Gaussian/Nongaussian Category
}

\author{
M. Selva Balan \\ Scientist d cwprs \& Research \\ Scholar,DIAT \\ Pune, India
}

\author{
C. R. S. Kumar, PhD \\ Associate Professor \\ Computer Engineering Division \\ DIAT, Pune, India
}

\author{
Swati D. Padole \\ PG Student \\ E\&TC Department \\ SCOE Pune, India
}

\begin{abstract}
In communication system, noise is very important factor. Like in free space, underwater noise is the result of many contribution. It is the combination of different noises which occur on surface or in deep water. Combination of all noises called as ambient noise which divide in two group i.e. manmade and natural noise. Acoustic signal is used to transmit the underwater data from one place to another place. Here focus on individual statistical analysis of all noises also done the simulation and from that decide, this is Gaussian or not. Generally underwater noise is additive with non-gaussian. Again focus on types of noises. In underwater, different noise occur at different frequency. For ship noise, ship activity is important parameter and for wind noise wind speed is more important. Here consider ship activity is medium and wind speed is $25 \mathrm{~m} / \mathrm{s}$. An algorithm based on non-gaussian approach allow to generate number of sequence of samples for noise realization and on the bases of kurtosis level decide the gaussianity. Again focus on combination of all noise like noise model and calculate the PSD of individual noise which indicate different noise occur at different frequency.
\end{abstract}

\section{Keywords}

Noise,Types of noise,statistical analysis, noise model.

\section{INTRODUCTION}

Communication is nothing but transfer of information from one place to another place. Now a day science and technology go beyond human expectation. Like free space, underwater technology also improve. Underwater communication not done by electromagnetic or optical signal, it is done by acoustic signal. Because these two signal is good for free space not for underwater communication, it provide lot of disadvantages during underwater. While acoustic wave provide lot of advantages like less attenuation, high reliability, travel long distance with low frequency only the lack of bandwidth is big issue with that signal. Acoustic signal is a sound signal. When underwater object vibrate they create sound presser wave that alternatively compressed \& decompressed water molecule as the sound wave travel through reservoir. The compression and decompression associated with sound wave are detected as change in pressure. The velocity of acoustic wave is $1500 \mathrm{~m} / \mathrm{s}$. It require elastic material for propagation (i.e. solid, liquid \& gas). This speed is depend on three factors such as temperature, salinity and depth. Speed can be calculated by

$$
\begin{aligned}
& C=1449.2+4.6 T-0.055 T^{2}+0.00029 T^{3}+(1.34-0.010 T)(S \\
& 35)+0.016 z
\end{aligned}
$$

Where, $\mathrm{c}$ is the sound velocity in $\mathrm{m} / \mathrm{s}$; $\mathrm{T}$ is temperature in ${ }^{\circ} \mathrm{C}$; $\mathrm{S}$ is salinity in p. s. $\mathrm{u}$. and $\mathrm{z}$ is depth in $\mathrm{m}$.

Temperature: Reservoir water temperature is decreases from the surface to the seabed. But there are many variation from this general trend. The time and space variability is maximal in the shallower layer but decrease with depth. Beyond a typical depth the average temperature remains stable and it is decrease very slowly with depth and varying very little from one place to other. According to temperature, sound is vary (that is on the surface temperature is more means speed of the signal is more. And in depth, temperature is less then speed of sound also less.

Depth: Hydrostatic pressure make the sound velocity increase with depth, because of variation in compressibility coefficient.

Salinity: Reservoir water is made up of mix of pure water and dissolved salt. Salt mass percentage define salinity and it measure in PSU. Salinity usually varies slightly with depth.

\section{NOISE}

Speaking about the underwater communication is the most challenging subject, because of variation. Day to day increase the industrial interest in offshore oil and gas extraction will affect the diversity in seawater. Man-made source is the most dominating source in ocean like ship noise. Different part of ship produce different noise among that propeller noise is most dominating. This environment create very complex noise in water called as ambient noise. It is the combination of natural and anthropic noise. Here data is to be collected from bathymetry which consist mixture of noise. Practical is to done at khdakawasla dam, Pune on $10^{\text {th }}$ September 2017 with single beam echo sounder having frequency $16 \mathrm{KHz}$ and depth is $200 \mathrm{~m}$ as shown in fig1.Data is to be recorded and simulate through matlab. Therefore PSD of different noises is givien as

$$
\begin{aligned}
& \mathrm{Nt}(\mathrm{f})=17-30 \log (\mathrm{f}) \\
& \mathrm{Ns}(\mathrm{f})=40+20(\mathrm{~S}-5)+26 \log (\mathrm{f})-60 \log (\mathrm{f}+0.03) \\
& \mathrm{Nw}(\mathrm{f})=50+7.5 \mathrm{w}+20 \log (\mathrm{f})-40 \log (\mathrm{f}+0.4) \\
& \mathrm{Nth}(\mathrm{f})=-15+20 \log (\mathrm{f})
\end{aligned}
$$

Where $\mathrm{f}$ is the frequency in $\mathrm{kHz}$. Therefore total noise PSD is

$$
\operatorname{Sxx}(f)=N t(f)+N s(f)+N w(f)+N \operatorname{th}(f)
$$


Here each noise is occur at different frequency. At low frequency turbulent noise is occur with having frequency from 0.1 to $10 \mathrm{~Hz}$. Ship noise occur at frequency $10 \mathrm{hz}$ to $100 \mathrm{hz}$.Wind noise occur at frequency $100 \mathrm{~Hz}$ to $100 \mathrm{Khz}$. This is generally occur on reservoir surface and its effect is depend upon wind speed. And the last is thermal noise which occur at frequency above the 100Khz.This is generated because movement of water molecule. It is occur at very high frequency. See fig 4 . Which indicate result of simulation of different noises.

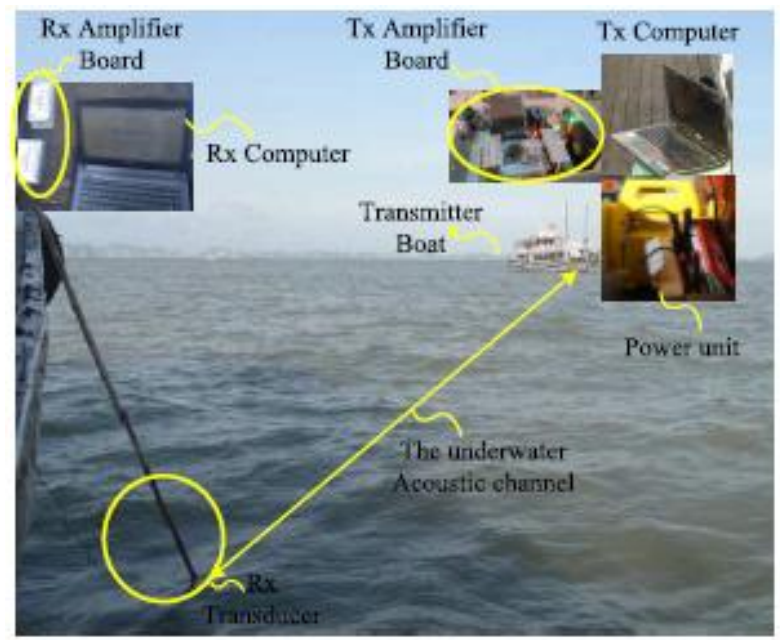

Fig 1. Scenario to collect acoustic data from reservoir.

\subsection{Wind noise:}

Wind noise is depend upon wind speed. If wind speed is high then breaking of waves occur and produce the sound. If we consider on shallow water then it is the combination of different noise. Generally this noise occur on surface of reservoir. And so because of that it is somewhat difficult to model. Wind blowing at a certain speed and from that source level (SL) can be obtained. Noise level by wind is given by

$\mathrm{NL}=\mathrm{A} 10 \mathrm{n} \log 10 \mathrm{u}$

Where A \& $\mathrm{n}$ are variable that may vary with frequency and location of measurement and $u$ is wind speed.

\subsection{Ship noise:}

Noise generated by ship is radiated by different part of ship. However the underwater noise spectrum is dominated by two main principal sources i.e. machinery and propeller. Propeller noise is the most dominating source which generate the noise on surface of sea. Rotating propeller create positive and negative pressure on water. This negative pressure create bubbles and produce the sound. Producing force on water is depend on the propeller blade. The propeller blades are rotating twisted wigs that produce the strong hydrodynamic force. This force create the noise which is occur at low frequency.

\subsection{Turbulent noise:}

Obstacle is come by flowing of river water and steam and create the turbulence as well as air bubble formation which produce the specific sound. This is nothing but background noise. In such condition signal to noise ratio is low and then difficulty is occur during the analysis of capture data. Second one is because of the turbulence, change in pressure is occur inside the turbulence region. This dynamically varying pressure is pickup by the pressure sensitive hydrophone located in turbulent region. If the turbulent component of flow is written as $\mathrm{u}$, then the associated dynamic pressure is qu2 where $\mathrm{q}$ is fluid density.

\subsection{Multipath:}

When the source transmit the signal toward the receiver then this signal is attenuated at surface or bottom means the signal is not come directly toward the receiver. This signal is arrive in different path with different time delay toward the receiver. This is nothing but the multipath signal. When the signal is arrive with different echo then the amplitude of the signal is decrease. Generally number of echoes or reflection of signal increase when propagation medium is small or limited. This reflection of signal is different in shallow and deep water. In shallow water normal mode theory is used to provide accurate result and in deep water ray theory is used. In high frequency at short distance multiple echo is created in the time domain. Whereas at low frequency, most of the time signal is stable.

$\mathrm{r}(\mathrm{t})=\mathrm{s}(\mathrm{t})+\mathrm{n}(\mathrm{t})$ with $\mathrm{s}(\mathrm{t})=\sum_{i=1}^{K}$ ai $e(t-\tau i)$

With an impulse response is

$\mathrm{H}(\mathrm{t})=\sum_{i=1}^{K} a i \delta(t=\tau i) \quad a i, \tau i € R$

Where ai is attenuation and $\tau i$ is time delay of ith path. $\delta($.$) Is$ delta function, $\mathrm{k}$ is number of path.

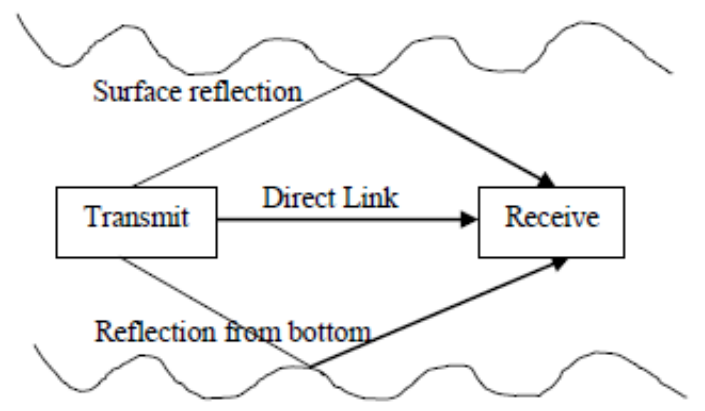

Fig 2. Multidimensional propagation.

\section{NOISE MODEL}

Here consider model which include all noises. Acoustic signal is used as input signal which is transmit in the form of sine wave with frequency $16 \mathrm{khz}$. As we know different types of noises occur at different frequency. Once the signal is transmit then start to add the noise. One by one noise is added and signal get corrupted. Initially multipath interference is occur because of that mean of input signal is change see in below table 1. Then step by step other noise is added like ship, thermal, wind and turbulence, see in below table 1 mean ans standard deviation (SD) is deflect when noise is added. Fig 3 indicate more than one noise added in signal. Fig 4 indicate the different types of noise occur at different frequency. At very low frequency turbulent noise is occur and at very high frequency thermal noise is occur. At moderate frequency ship and wind noise is occur. 


\section{STATISTICAL ANALYSIS}

Statistical analysis means do individual analysis of each noise and calculate all parameter. Acoustic noise is the sequence of samples which having random charactertics. Since the simulating the noise based on Gaussian process. Generally the underwater noise is far from the gaussian. Normal distribution characterized the mean is zero, variance is one and level of kurtosis is 3[4]. The values of kurtosis of random variable indicate how far it's PDF from gaussian shape. Generation of noise is focused to produce the sequence of random variable with lower or higher kurtosis than 3. This level of kurtosis indicate the gaussianity and any shift from this value indicate the non- gaussian shape. See table1, how kurtosis value for every noise is change which indicate noise is non gaussian. Skewness value is indicate the symmetry property. If Skewness value is 0 then it is more symmetry. If its value is negative then signal is shift negative side. And if its value is positive then signal is shift toward positive side. Its value should be less than 1.9.

\section{RESULT}

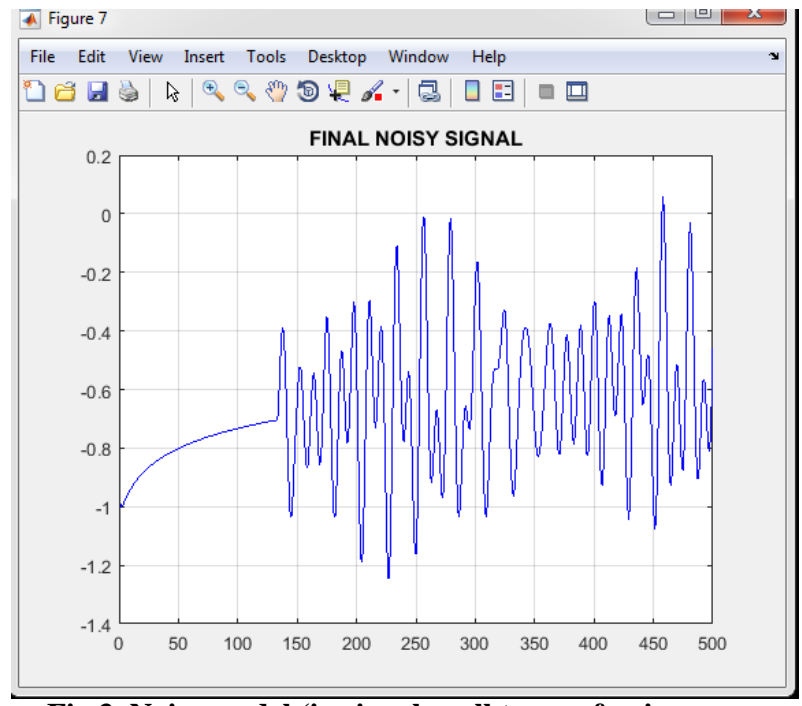

Fig 3. Noise model (i.e. involve all types of noise mean turbulence, wind, ship and thermal noise.)

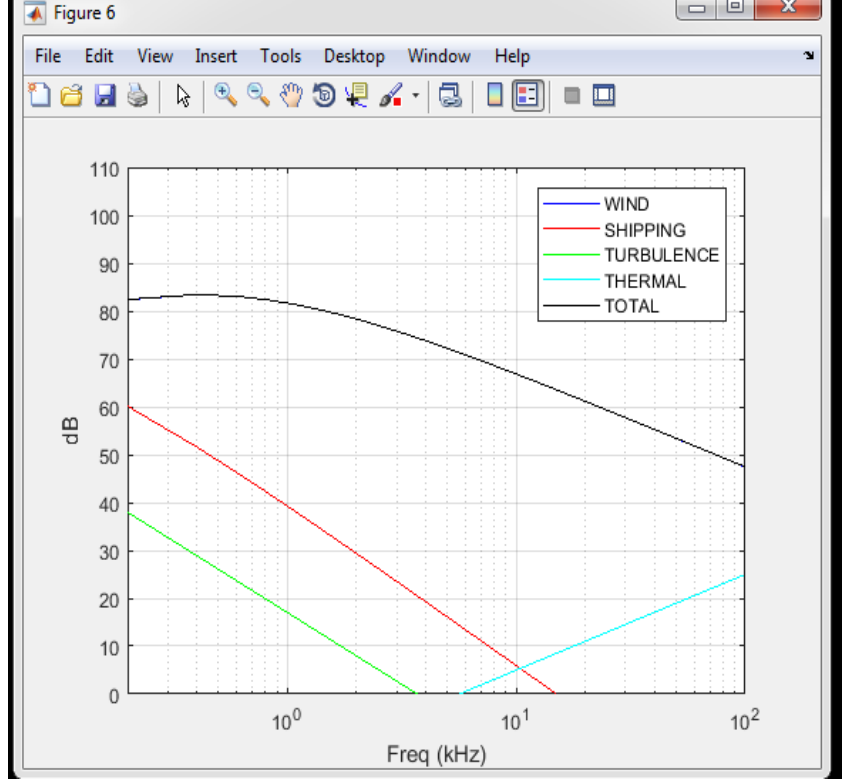

Fig 4. The noise power spectrum level of underwater noises which indicate different noise occur at different frequency.

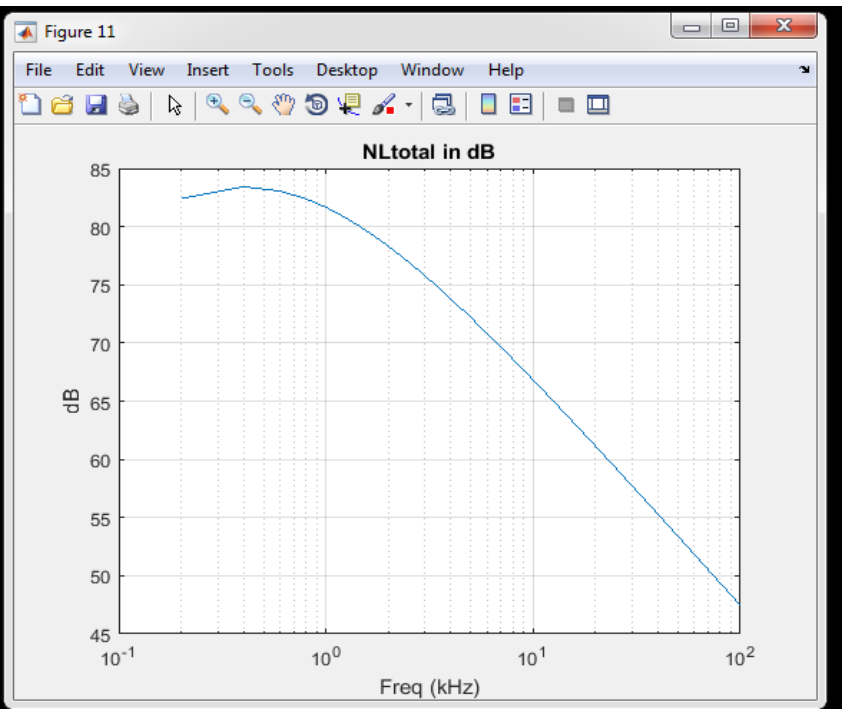

Fig 5. Total noise signal in $\mathrm{db}$ 
Table 1. Statistical parameter of signal and noises

\begin{tabular}{|l|l|l|l|l|l|l|}
\hline Parameter & $\begin{array}{l}\text { Input } \\
\text { signal }\end{array}$ & $\begin{array}{l}\text { Input+ } \\
\text { Multipath } \\
\text { noise }\end{array}$ & $\begin{array}{l}\text { Input+ } \\
\text { thermal } \\
\text { noise }\end{array}$ & $\begin{array}{l}\text { Input+ } \\
\text { wind } \\
\text { noise }\end{array}$ & $\begin{array}{l}\text { Input+ } \\
\text { Ship } \\
\text { noise }\end{array}$ & $\begin{array}{l}\text { Input+ } \\
\text { Turbulent } \\
\text { Noise }\end{array}$ \\
\hline Mean & 0.309546 & -0.001309 & 0.006225 & 55.707983 & 13.40509 & -30.07608 \\
\hline SD & 35.357267 & 0.212213 & 0.711845 & 7.661346 & 14.28574 & 12.715423 \\
\hline Variance & 1252.966 & 0.045034 & 0.506723 & 58.696224 & 204.0825 & 161.681994 \\
\hline Kurtosis & 1.500 & 3.670681 & 1.500924 & 4.560780 & 6.663944 & 6.993309 \\
\hline Skewness & -0.013136 & 0.187012 & 0.013136 & 1.369534 & 1.739109 & 1.786266 \\
\hline
\end{tabular}

\section{CONCLUSION}

In communication system noise play a very important role. Acoustic noise in the reservoir come from many sources. Surface agitation and ship noise is the most dominant source of ambient noise. Here we done the statistical analysis and on the basis of that we differentiate Gaussian or non-gaussian noises, basically we find out from kurtosis value. Kurtosis values is not 3 means this noise is non-gaussian. From that we can say that underwater noise is non gaussian noise. We have also simulate the effect of noise in the input signal using the additive noise model by adding all five types of noise independently and calculate statistically.

\section{ACKNOWLEDGMENT}

We are thankful to national irrigation center Kerala team for providing the data of reservoir in India. I would like to express my special thanks \& gratitude to Shini KK. Dy Director, KERI, Peechi, Kerala as well as our guide Dr. R.S.Kawitkar, Prof, Dept. of electronic, Sinhgad College of engineering, Pune who gave me the golden opportunity to do this wonderful project, which also helped me in doing a lot of research and I came to know about so many new things I am really thankful to them.

\section{REFERENCES}

[1] Andrew C. Singer, Jill K. Nelson Suleyman S. Kozat, "Signal Processing for Underwater Acoustic Communications", IEEE Communications Magazine • January 2009.

[2] ChiYen Nguyen*, Ha Viet Dot, Van Due Nguyen+ and Helio Augusto, "Underwater ambient noise model and verification in the underwater OFDM system", Muzamane KICS-IEEE International Conference on Information and Communications with Samsung LTE \&SG Special Workshop 978-15386-0962-0/17/\$31.00 @2017 IEEE.

[3] Federico Traverso, Tomaso Gaggero, Giorgio TaniMichele Viviani, Diego Villa, Stefano Gaggero. "STATISTICAL ANALYSIS OF SHIP NOISE RECORDS".
[4] F.Traverso and G. Vernazza, A.Trucco, "Simulation of non-white and non-gaussian underwater ambient noise".

[5] Gu Xiang-ping*a, Yang yana, Hu Rong-lina, "Analyzing the Performance of Channel in Underwater Wireless Sensor Networks(UWSN), 2011 Published by Elsevier Ltd.

[6] Michel Bouvet, Stuart C. Schwartz, "Underwater noises: Statistical modeling, detection, and normalization".

[7] Nejah NASRI Abdennaceur KACHOURI Laurent ANDRIEUX Mounir SAMET, "Design considerations for wireless underwater communication transceiver", 978-1-4244-26287/08/\$25.00 @2008 IEEE.

[8] Nima Bahrami, Nor Hisham Haji Khamis, Ameruddin Baharom, "Evaluation of Underwater Acoustical Intermittent Ambient Noise Sea surface Biological Traffic noise", 2015 IEEE, $11^{\text {th }}$ International Colloquium on Signal Processing \& its Applications (CSPA2015)", 6 -8 Mac. 2015, Kuala Lumpur, Malaysia.

[9] Sharbar Banerjee1, Monika Agrawal, "Underwater Acoustic Noise with Generalized Gaussian Statistics: Effects on Error Performance", 978-14799-0002-2/13/\$31.00 @2013 IEEE.

[10]Tri Budi Santoso, Endang Widjiati, Wirawan, Gamantyo Hendrantoro, "Ambient Noise Measurement and Characterization of Underwater Acoustic Channel in Surabaya Bay", 2015 IEEE Asia pacific conference on wireless and mobile. 